\title{
Vida y muerte de dos mujeres de Brigantium (NW de Iberia) mediante isótopos estables y antropología forense
}

GRANDAL-D'ANGLADE, A. ', SERRULLA RECH, F. 2, TOMAS BOTELLA, V. 3, PÉREZ RAMA, M ${ }^{1}$, GOMEZ, M. ${ }^{2}$ e RAMIL GONZALEZ, E. ${ }^{3}$

(1) Universitario de Xeoloxía. Universidade da Coruña. Edificio de Servicios de Investigación. Campus de Elviña s/n. 15071 A Coruña.xeaurora@udc.es; mperezr@udc.es

(2) Unidad de Antropología Forense. Instituto de Medicina Legal de Galicia. Hospital de Verin 32600 Verin (Ourense). fernandoserrullarech@hotmail.com

(3) AXA Arqueoloxía S.C. Avenida dos Caídos 3-7ª.15009 A Coruña. vtomasbotell@gmail. com; emilioramilgonzalez@gmail.com.

\section{Resumen:}

En este trabajo se obtienen datos antropológicos e isotópicos a partir de los restos óseos de dos individuos de edad tardorromana a efectos de reconstruir sus condiciones de vida y las causas de su muerte. Se trata de dos esqueletos femeninos conservados en el Museo Arqueolóxico de A Coruña datados entre los siglos III a VI dC. Se han aplicado técnicas de antropología forense para determinar patologías y lesiones que puedan haber afectado a los individuos estudiados. Para el estudio de paleodieta, se ha extraído colágeno óseo que se analiza mediante espectrometría de masas de relaciones isotópicas. Las relaciones isotópicas de ${ }^{13} \mathrm{C} /{ }^{12} \mathrm{C}$ y ${ }^{15} \mathrm{~N} /{ }^{14} \mathrm{~N}$ obtenidas se comparan con datos procedentes de otras poblaciones contemporáneas. Los perfiles antropológicos confirman que se trata de mujeres adultas jóvenes, de posible ancestralidad caucásica, con signos de hiponutrición e infección crónica y cuyas señales isotópicas del colágeno muestran una importante contribución de los moluscos en su dieta. Estos resultados concuerdan con los datos arqueológicos disponibles, reflejando el progresivo empobrecimiento de la ciudad durante la crisis del Bajo Imperio. 


\title{
PALABRAS CLAVE:
}

Paleodieta, isótopos estables, colágeno óseo, antropología Forense, Bajo Imperio Romano, Brigantium, A Coruña.

\begin{abstract}
:
In this paper we obtain anthropological and isotopic data from the skeletal remains of two individuals of the late Roman age in order to rebuild their lives and causes of death. These are two female skeletons preserved in the Archaeological Museum of A Coruña dating from III to VI centuries AD. Forensic anthropology techniques have been applied to identify diseases and injuries that may have affected the individuals studied. For the palaeodiet study, extracted bone collagen was analysed by isotope ratios mass spectrometry. The ${ }^{13} \mathrm{C} /{ }^{12} \mathrm{C}$ and ${ }^{15} \mathrm{~N} /{ }^{14} \mathrm{~N}$ ratios are compared with data from other contemporary populations. Anthropological profiles confirm that these are two young adult females of Caucasian ancestry, with signs of chronic malnutrition and infection. Their isotopic signature shows a significant contribution of molluscs in their diet. These results are consistent with the available archaeological data, reflecting the progressive impoverishment of the city during the crisis of the Lower Empire.
\end{abstract}

\section{KEY WORDS:}

Palaeodiet, stable isotopes, bone collagen, forensic anthropology, Lower Roman Empire, Brigantium, A Coruña. 


\section{INTRODUCCIÓN}

\subsection{El marco geográfico: la ciudad de Brigantium}

La ciudad de Brigantium, actualmente A Coruña, se encuentra situada en el denominado Golfo Ártabro del NW de la península Ibérica (Figura 1). Situada sobre un amplio promontorio rocoso unido a tierra firme por un istmo antaño cubierto de arena, a lo largo de su historia temprana la ciudad experimentó una expansión y una retracción paralelas al sino del Imperio.
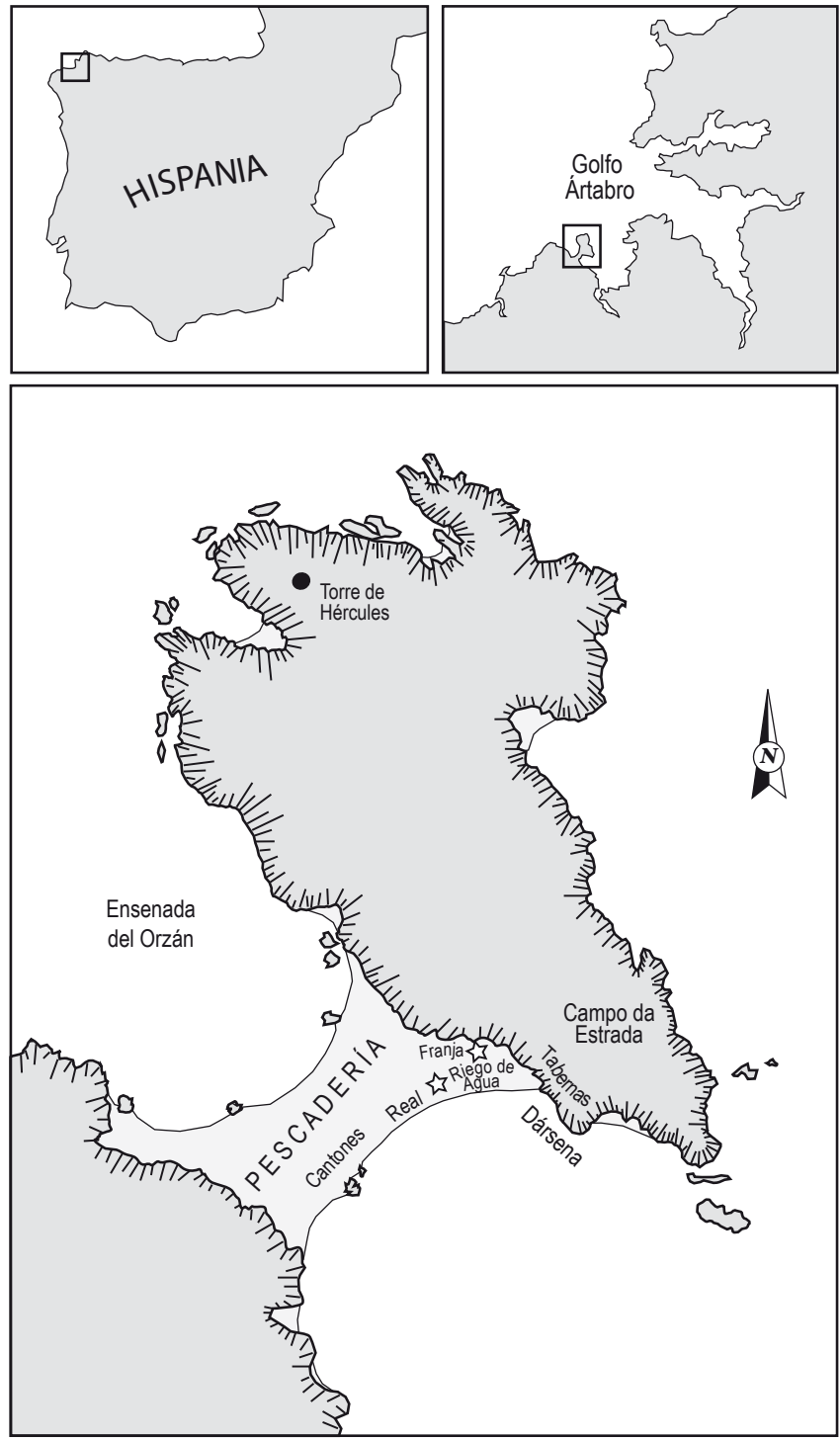

Fig. 1. Mapa de A Coruña en su aspecto aproximado de los primeros siglos de nuestra Era, donde se muestra la ubicación de las tumbas de las que proceden los esqueletos estudiados y otros lugares mencionados en el texto. 
Ciudad portuaria que fue mansio de la Vía XX per loca maritima del itinerario Antoniano, comunicaba directamente con Lucus Augusti (Lugo), capital del conventus, y con otras capitales como Bracara Augusta y Asturica Augusta, sirviendo como puerto de indudable interés en las rutas comerciales al abrigo de las borrascas atlánticas. Dion Cassio, en su Historia Romana (Libro XXXVII, 52-53) menciona la llegada de Julio César a la población, en el año 61 a. C. La ciudad romana comienza a ser importante en la época Flavia, a finales del siglo I y durante el siglo II d. C., cuando se construye el Faro conocido como "Torre de Hércules", aún en funcionamiento y declarado por la UNESCO Patrimonio de la Humanidad. Se ha propuesto también que el crecimiento urbano respondió no sólo a la actividad comercial sino a haber servido de puerto base para el avituallamiento de las legiones destacadas en Britania (Bello Diéguez, 2009; López Pérez, 2010a). Durante el siglo IV Brigantium debió de seguir siendo un puerto importante en las rutas del imperio. Es mencionada por Paulo Orosio (Historice adversvm Paganos) como uno de los tres ángulos de Hispania, pues considera que la forma de la península es triangular, siendo uno de sus vértices Brigantium, que "levanta en la dirección de Bretaña un altísimo faro". A partir del siglo IV, sin embargo, la ciudad se retrae, hasta el punto de desaparecer de las crónicas escritas durante varios siglos (Naveiro López, 1994; Bello Diéguez, 1995).

\subsection{Objetivos del trabajo}

Este estudio tiene su origen en la iniciativa del Ayuntamiento de la ciudad de A Coruña de poner rostro a los restos humanos más antiguos conservados de la ciudad, con motivo del $8^{\circ}$ centenario de la misma. Para ello, se estudiaron desde el punto de vista antropológico tres esqueletos humanos de época romana. Conservados en el Museo Arqueolóxico de Coruña, proceden de diversas excavaciones arqueológicas desde el año 1949 y conservan suficientemente la morfología craneofacial para ponerles rostro mediante técnicas de reconstrucción facial y reconstruir su vida y su muerte mediante análisis antropológicoforenses (Serrulla \& Gómez, 2008).

El objetivo principal de este trabajo consiste en aportar nuevos datos sobre estos individuos relacionados con su estilo de vida. Se trata del estudio de la paleodieta de estos humanos mediante el análisis de isótopos estables del colágeno óseo. Este análisis es una herramienta ampliamente utilizada para obtener inferencias ecológicas de los mamíferos extintos (dieta, fisiología, etc.) proporcionando además datos paleoambientales sobre el medio físico donde vivieron (Tieszen y Bouton, 1989; Koch, 1998; Hedges et al., 2004). Entre los más utilizados se encuentran los isótopos del carbono y del nitrógeno contenidos en el colágeno preservado en el tejido óseo.

En los últimos años se han empleado insistentemente en el estudio de la composición de la dieta humana en el pasado, siendo cada vez más abundantes los datos de Neandertales, de humanos modernos paleo y mesolíticos, de yacimientos Neolítico o de distintos pueblos de épocas ya históricas (Bronce, Hierro, romanización, edad media, etc.). Mediante estos estudios se han determinado unos patrones de alimentación cambiantes a lo largo del tiempo (Lubell et al., 1994; Schoeller, 1999; Richards, 2002; Tykot, 2003; Hedges \& Reynard, 2007) que serán discutidos más adelante. 


\subsection{El contexto arqueológico.}

Las actuaciones arqueológicas de las que provienen los esqueletos fueron realizas en un contexto de arqueología urbana, con motivo de la ejecución de obras civiles: renovación del empedrado de la calle Real en el caso de los exhumados en el año 1949 por J. María Luengo (Luengo, 1955) y rehabilitación de un inmueble en la misma vía (Tomás Botella, 2004), por lo que la extracción de los restos se ha producido en ambos casos en un entorno muy alterado por la dinámica histórica urbana y en una extensión superficial limitada artificialmente por un parcelario más o menos reciente, que poco tiene que ver con la realidad espacial y topográfica de la ciudad romana, registrándose los datos de forma fragmentaria, lo que dificulta su interpretación y contextualización cultural. A ello hay que añadir la precariedad de medios y escasez de tiempo con que se realizan las actuaciones por las propias circunstancias de trabajo, que debe ejecutarse con entendimiento de intereses ajenos a su carácter patrimonial.

A esto hay que añadir, en este caso, las dificultades concretas que presenta la composición del subsuelo en el sector de la Pescadería de A Coruña, dado que se asienta sobre el istmo arenoso que une a tierra firme el promontorio de la Ciudad Vieja, con presencia de un substrato muy homogéneo que borra la información cronológica que aportan las deposiciones de carácter antrópico de mayor antigüedad, no pudiéndose calibrar con la suficiente exactitud, según la lectura estratigráfica, el momento en que se abren las fosas de inhumación. Por lo tanto, las discriminaciones cronológicas se deben hacer, necesariamente, en base a otros criterios de tipo relativo y a los aspectos culturales que puedan ser documentados. Sin embargo ese mismo sustrato arenoso puede haber favorecido o incluso permitido la preservación de los restos óseos gracias a su pH básico.

\section{MATERIAL}

\subsection{Esqueletos MAC-110 y MAC-108}

Los esqueletos llamados MAC-110 y MAC-108 se encuentran en el Museo Arqueológico desde hace más de 50 años. No se conservan datos concretos de su localización, aunque ambos son parte del conjunto de cinco esqueletos de adulto y uno infantil hallados en la calle Real por Luengo en sus excavaciones de 1949.

Según este autor (Luengo, 1955) el 3 de marzo de 1949 frente al $n^{\circ} 9$ de la calle Real se hallan los esqueletos de un hombre, una mujer y un niño. Consta la realización de un estudio antropológico por el Dr. Peña que acredita un traumatismo craneal (occipucio) en el caso de la mujer. A mediados del mes de marzo de 1949, en una excavación dirigida por él mismo, se hallaron frente al número 7 de la misma calle huesos humanos $\mathrm{y}$ trozos de tégulas procedentes de una sepultura destruida en un nivel revuelto por los antiguos tendidos eléctricos. En la zona denominada "frente al Salón París", correspondiente al $\mathrm{n}^{\circ} 1$ de la citada calle, el dia 5 de mayo del mismo año se descubrió una cista antropoide 1,70 m (L), 0,50 m (cabecera) y 0,45 m (pies) que contenía un esqueleto. En las proximidades se hallaron huesos de animales y conchas de berberechos, mejillones y almejas. En las proximidades de la casa $n^{\circ} 1$ de la calle Real, en una cista de piedras cubierta cuidadosamente con 
lajas de granito, se halla otro esqueleto en postura supina con los brazos extendidos. La cabeza había desaparecido, cortada por los cimientos de la calle Real. Delante de las casas números 54 a 60 de la calle Riego de Agua (continuación de la Calle Real) se descubrió una sepultura formada por piedras de granito y tapada con lajas del mismo material. De lecho antropoide de 1,90 $\mathrm{m}$ de larga, $0,40 \mathrm{~m}$ de cabecera y $0,22 \mathrm{~m}$ en los pies, el esqueleto se encontraba colocado en decúbito supino con los brazos cruzados sobre el vientre. Junto a la rodilla derecha del esqueleto en posición tumbada había una pequeña vasija fusiforme. La posición de todos los esqueletos estaba orientada de Este-Oeste, con la cabecera a poniente.

Este mismo autor (Luengo, 1955) data la necrópolis entre los siglos I y IV d.C. Las inhumaciones objeto del artículo, por el rito empleado, se centran en un período bajo imperial romano (siglos III a VI d.C.). Los enterramientos estudiados, que se pueden asociar a tumbas arquitectónicas según lo indicado por Luengo, pueden ser datados en el período entre los siglos IV a VI d.C., conforme a la revisión que de esta excavación hizo el arqueólogo Juan Naveiro (Naveiro López, 1988).

De esta zona se estudiaron dos individuos, que se seleccionaron por encontrarse relativamente completos y en un estado de conservación aceptable. El esqueleto MAC110 conserva el cráneo en buen estado. El esqueleto poscraneal presenta buen estado de conservación, aunque se observa la falta de algunos huesos (cúbitos, escápulas, muchos huesos de ambas manos y coxal y fémur izquierdos). Los huesos contienen arena adherida que despega con el dedo y muchos presentan una peculiar pátina blanquecina.
En cuanto al esqueleto MAC-108, su cráneo se halla en buen estado de conservación, blanqueado, fragmentado pero adherido, con ausencia de paladar (maxilar y palatinos), etmoides y parte del esfenoides. El esqueleto poscraneal se halla en buen estado de conservación, blanqueado y sujeto mediante alambres que perforan los huesos a un tablero. Se realizó estudio antropológico (Serrulla \& Gómez 2008), pero el hecho de que su esqueleto haya sufrido un tratamiento químico de blanqueado podría haber supuesto un problema a la hora de extraer colágeno bien preservado, por lo que de este individuo no se realizó análisis isotópico.

\subsection{Esqueleto MAC-4414}

Los restos del esqueleto llamado MAC4414 se hallaron en la excavación realizada en el solar de la calle Real n ${ }^{\circ} 34$ de A Coruña entre los años 2000 y 2004, según se indica en la Memoria Arqueológica elaborada por el Arqueólogo Víctor Tomás Botella y la empresa arqueológica AXA Arqueoloxía. Se trata de una necrópolis romana de incineración datada en torno al siglo II, sobre la que se superpone la inhumación que nos ocupa, de edad en torno a los siglos III y IV d.C.. Los restos recibieron en la Memoria Arqueológica la denominación 'INHUMACION No 3 '.

Para el esqueleto MAC-4414 habría que pensar quizá en el mismo intervalo cronológico que para los individuos anteriores, aunque al presentarse en fosa simple, sin ajuar, mostrando un empobrecimiento de los ritos funerarios, sería relativamente posterior. No convendría interpretar esta distinción como fruto de diferencias sociales, sino en cambios 
culturales, que podrían llevarnos hasta época medieval, otro momento superpuesto al anterior en esta necrópolis de la calle Real, aunque no sea descartable, por paralelismos, su datación tardorromana.

En general el estado de conservación de los restos del individuo MAC-4414 es aceptable. El cráneo mantiene un buen estado de conservación, pero el esqueleto poscraneal muestra deterioro marcado en las epífisis de los huesos largos, columna vertebral y pelvis. En torno al orificio occipital existe pérdida posmortem de occipital, esfenoides y parte de ambos peñascos. El vómer está incompleto posmortem.

\section{MÉTODOS}

Para los estudios isotópicos, sobre todo para los encaminados a la determinación de la paleodieta, se emplea generalmente el colágeno preservado en el tejido óseo. Al tratarse de una proteína, el colágeno registrará los valores isotópicos procedentes de la alimentación y del correspondiente fraccionamiento que se produce durante la asimilación de las sustancias nutritivas y la síntesis del propio colágeno. Su tasa de renovación es de varios años, por lo que su estudio permite reconstruir la dieta del individuo durante un largo período de su vida.

A los efectos de obtener muestra para estudio de paleodieta, se emplearon restos de hueso cortical de MAC-110 y MAC-4414 en cantidad total de $350 \mathrm{mg}$. Las muestras se lavan en cubeta de ultrasonidos en sucesivos baños de agua destilada y acetona, y posteriormente se pulverizan en mortero de agata. Del polvo de hueso obtenido se destinan unos $20 \mathrm{mg}$ a un análisis elemental de contenido en $\mathrm{C}$ y $\mathrm{N}$ expresado en porcentaje del peso total. Estos datos son buenos indicadores de la recuperabilidad del colágeno preservado (Bocherens et al. 2005a). El análisis se realiza en un analizador elemental ThermoFinnigan Flash EA1112 con precisión analítica mejor que el $0,1 \%$.

La extracción del colágeno se basa en el método propuesto por Bocherens et al., 1997. Consiste en someter el polvo de hueso a una serie de digestiones en ácidos y bases que eliminan la materia inorgánica, seguidas cada una de ellas por ultrafiltraciones que eliminan las moléculas de menor tamaño, para obtener así el colágeno óseo, una proteína de gran tamaño formada por tres cadenas de polipéptidos organizadas en triple hélice con una composición aminoacídica bastante simple y repetitiva (glicina, prolina e hidroxiprolina principalmente no siendo ninguno de ellos esenciales, es decir, no deben ser incorporados en la dieta pues pueden ser sintetizados). El colágeno extraído en forma de gelatina, se liofiliza para su posterior análisis elemental e isotópico. Éste se realizó en los Servicios de Apoio á Investigación de la Universidade da Coruña, en un Analizador elemental EA1108 (Carlo Erba Instruments) acoplado mediante una interfase ConfloIII (ThermoFinnigan) a un espectrómetro de masas de relaciones isotópicas MAT253 (ThermoFinnigan), con reproducibilidad analítica mejor que $0,2 \%$ para el $\mathrm{C}$ y el $\mathrm{N}$, utilizando los estándares habituales (PDB para el carbono y AIR para el nitrógeno). Las medidas realizadas dan información de la abundancia isotópica relativa de cada isótopo con respecto a su estándar, ya que el equipo dispone de doble entrada. Además, se miden los porcentajes de $\mathrm{C}$ y $\mathrm{N}$ en el colágeno, que serán fundamentales a la hora de determinar la calidad del colágeno extraído. 
La técnica se basa en el hecho de que en la naturaleza existen diversos elementos que se presentan con distinto número de neutrones y por lo tanto, distinta masa atómica. Estos isótopos son trazadores paleobiológicos pues, como resultado de la diferencia en masa atómica, muestran diferentes propiedades termodinámicas $\mathrm{y}$ cinéticas. En aquellos elementos con masa atómica $<40$ tales diferencias pueden llevar a un fraccionamiento isotópico (un cambio en la abundancia relativa de cada isótopo) durante los procesos fisicoquímicos que intervienen en las reacciones metabólicas, lo cual se traduce en una diferente proporción entre isótopo pesado e isótopo ligero antes y después del proceso (Koch, 1998).

El fraccionamiento natural es bajo, de forma que las proporciones isotópicas se miden en tantos por mil (\%o) de desviación frente a un estándar, usando la notación $\delta$ que refleja la diferencia en la proporción entre isótopo pesado y ligero de la muestra con respecto al estándar.. Los valores positivos de $\delta$ indican un enriquecimiento en el isótopo pesado con respecto al estándar, mientras que los valores negativos muestran una disminución de su abundancia relativa. El estándar del carbono está muy enriquecido en el isótopo pesado, de manera que en comparación, tanto los vegetales como los animales e incluso el $\mathrm{CO}_{2}$ atmosférico presentan valores negativos de $\delta^{13} \mathrm{C}$.

\section{RESULTADOS Y DISCUSIÓN}

\subsection{Aportación del estudio antropológico}

Un estudio previo de los restos óseos mediante métodos clásicos de antropología forense (Serrulla \& Gómez, 2008) permitió la determinación de sexo, edad, talla, identidad racial, lesiones y enfermedades de los individuoas estudiados, así como una reconstrucción facial de los mismos. Partiendo de ese estudio, desde el punto de vista antropológico se destacan los siguientes datos de interés:

\subsubsection{Individuo MAC-110.}

El esqueleto pertenece a una mujer adulta de una edad estimada en torno a 20-25 años, de talla sobre los $160 \mathrm{~cm}$ y con caracteres de ancestralidad caucásica (raza blanca). El ángulo del perfil facial se corresponde con una identidad racial negroide (Reverte Coma, 1991), lo que permite considerar la hipótesis de que la joven pudiera tener un origen norteafricano.

No se han hallado evidencias relativas a la causa de la muerte. Existen no obstante algunos datos que permiten reconstruir la patobiografía de esta joven mujer.

El esqueleto presenta patologías en las vértebras denominadas 'hernias de Schmörl', que representan una fase inicial de la hernia discal. El núcleo del disco intervertebral se hunde en el hueso y deja un orificio en el hueso seco. En generalla aparición de hernias discales está relacionado en su génesis con el esfuerzo crónico al que se somete a la articulación intervertebral, aunque existen factores innatos y otros adquiridos. Es posible por lo tanto que una joven de 20 a 25 años que presente signos de hernias discales incipientes haya realizado esfuerzos físicos (carga de pesos) importantes (Campillo, 1983, 1993; Dastugue y Gervais 1992).

Otro rasgo de interés que se ha observado es la presencia de líneas de Harris, lo que se relaciona con un problema de falta de alimentación. Su existencia hace pensar en 
un estado carencial (Ortner, 2003; Pérez, 1995).

El esqueleto presenta lesiones en ambas tibias (Serrulla \& Gómez, 2008, Fig. 15), compatibles con varios diagnósticos y que presentan claramente distintas fases evolutivas. La lesión de la tibia derecha es claramente una lesión menos evolucionada que el resto y presenta signos de leve crecimiento perióstico por debajo, lo que sugiere que éste crecimiento perióstico se produjo días antes de la muerte (Serrulla, 2001). Estas lesiones pueden ser debidas a varias causas de las que se sugieren las siguientes: a) Osteomielitis: Es posiblemente el origen más probable de éstas lesiones. Puede tratarse de una infección diseminada por vía sanguínea de un foco situado en partes blandas (pulmón quizás) (Ortner, 2003). b) Sífilis secundaria: Las lesiones de la sífilis tienen una morfología similar y se localizan preferentemente en las tibias, pero se acompañan de otras lesiones en otros huesos y además son lesiones que raramente se presentan en personas jóvenes (Mann y Hunt, 2005). c) Cáncer y metástasis: El cáncer de mama y otros cánceres pueden avanzar y producir metástasis en los huesos (costillas, columna y piernas). Las lesiones son compatibles con una metástasis cancerosa aunque no hemos hallado lesiones en otras localizaciones. Las lesiones también son compatibles con la presencia de varios carcinomas que asientan en esta zona y muestras caracteres similares (zonas de osteolisis y zonas blásticas): condrosarcoma, sarcoma de Ewing, osteosarcoma, entre otros (Ortner, 2003).

En síntesis, la joven mujer que estudiamos es posible que realizara muchos esfuerzos físicos, que padeciera problemas de nutrición (ya fuera estado carencial o enfermedad crónica) y que su muerte estuviera relacionada con un proceso infeccioso grave que pudo acabar con su vida.

\subsubsection{Individuo MAC-4414}

El esqueleto estudiado pertenece a una mujer, adulta de una edad estimada en torno a 35 años, de talla sobre los 145 $150 \mathrm{~cm}$ y con caracteres de identidad racial caucásica (raza blanca). Como ocurre en el caso de MAC-110, existe un parámetro antropométrico (Indice Gnático de Flower) que se corresponde con una identidad racial negroide. Ello nos permite considerar como en el anterior la hipótesis de que la joven estudiada pudiera tener un origen norteafricano (Janz et al., 1994).

No se han hallado evidencias relativas a la causa de la muerte. Existen no obstante muchos datos que sugieren los siguientes datos patobiográficos:

El estudio del cráneo confirma que este individuo sufrió bastantes meses antes de la muerte un traumatismo cráneoencefálico en la zona frontal izquierda. El traumatismo produjo pérdida de hueso en su capa más externa y generó bordes nítidos, lo que nos sugiere que tuvo que producirse con un objeto corto-contundente de mayor componente contusivo que cortante. Podría tratarse de una azada, o de un cuchillo grande o espada pequeña. Este tipo de traumatismo es probable que hubiera curado con secuelas morfológicas (cicatrices) pero sin secuelas funcionales (sin repercusión neurológica encefálica) (Reichs, 1998).

En el maxilar inferior derecho se observa un avanzado foco de osteítis con fistulización, así como la existencia de una avanzada enfermedad periodontal para la edad estimada (Serrulla \& Gómez, Fig. 18). Ello 
nos confirma la presencia de un importante foco de infección oral activo en el momento de la muerte. Además hemos objetivado la presencia de una sinusitis crónica en el seno maxilar derecho con fistulización oral y escasos signos de regeneración de los bordes de la fístula oral. Ello permite considerar la extensión del foco de infección al maxilar superior (Nossintchouck, 1991).

Otro rasgo de interés es la hipotrofia de la extremidad superior izquierda (clavícula, humero, cubito y radio) que sugiere algún tipo de parálisis periférica por lesión medular o radicular.
En síntesis, la mujer cuyo esqueleto fue hallado en el solar de la calle Real de A Coruña padecía posiblemente desde bastante tiempo antes de morir, una forma incompleta de parálisis del brazo izquierdo (paresia). Meses antes de morir sufrió un fuerte golpe en la cabeza con algún instrumento corto-contundente tipo azada. Y también sufrió una avanzada infección crónica en la boca, dolorosa y activa posiblemente en el momento de la muerte. Es posible que la causa de la muerte tenga que ver con el importante proceso infeccioso de la boca (Ortner, 2003).

\begin{tabular}{|c|c|c|c|c|c|c|}
\hline Muestra & rendimiento & $\% \mathbf{C}$ col & $\% \mathbf{N}$ col & $\mathbf{C} / \mathbf{N}$ at & $\delta \mathbf{1 3 C}$ col & $\delta \mathbf{1 5 N}$ col \\
\hline MAC-110 & 8,3 & 41,0 & 14,8 & 3,2 & $-18,7$ & 10,2 \\
\hline MAC-4414 & 6,2 & 40,0 & 14,6 & 3,2 & $-16,7$ & 10,8 \\
\hline
\end{tabular}

Tabla 1. Resultados del análisis isotópico del colágeno óseo de MAC-110 y MAC-4414. El rendimiento de la extracción se expresa en miligramos de colágeno por gramo de hueso. $\% \mathrm{C}$ col y $\% \mathrm{~N}$ col son los porcentajes de $\mathrm{C}$ y $\mathrm{N}$ en el colágeno. $\mathrm{C} / \mathrm{N}$ at es la relación atómica entre $\mathrm{C}$ y $\mathrm{N}$. Valores de $\delta 13 \mathrm{C}$ (referidos al estándar PDB) y $\delta 15 \mathrm{~N}$ (referidos al nitrógeno atmosférico) se expresan en \%o.

\subsection{Estudio de paleodieta mediante análisis de isótopos estables}

\subsubsection{Calidad de la extracción}

Los resultados del análisis isotópico realizado a las muestras óseas de los individuos MAC-110 y MAC-4414 se presentan en la Tabla 1. En los dos individuos estudiados los valores de $\delta^{15} \mathrm{~N}$ y $\delta^{13} \mathrm{C}$ varían ligeramente, pero se encuentran dentro de los límites normales para la especie humana.

Resulta fundamental poder constatar que el colágeno esté bien preservado sin contaminaciones o pérdida de alguno de sus constituyentes para que el resultado del análisis isotópico sea válido. Lógicamente, el hueso fósil no conserva su colágeno intacto, sino que siempre sufre pérdidas, pero interesa determinar si esas pérdidas pueden falsear los valores isotópicos. También es necesario saber si el proceso de extracción de colágeno se ha realizado con éxito. Para comprobar la calidad del colágeno extraído se aplican tres criterios de calidad:

a) El rendimiento de la extracción. Se mide en miligramos de colágeno por gramo de hueso, generalmente expresado como porcentaje. El hueso fresco contiene aproximadamente un $22 \%$ de colágeno, pero para el hueso fósil se aceptan proporciones inferiores, hasta un límite de 3,5\% (Van Klinken, 1999). El método de extracción 
utilizado en este trabajo ofrece en ambos casos valores superiores al valor límite.

b) Las proporciones de carbono $\mathrm{y}$ nitrógeno en el colágeno extraído. Los valores medios en hueso fresco son de aproximadamente un $35 \%$ de $\mathrm{C}$ y entre 11 y $16 \%$ de N. Para los fósiles se aceptan porcentajes no inferiores al 13\% para el C y al 5\% para el N (Ambrose, 1990; De Niro, 1985). Algunos autores prefieren considerar sólo aquellas muestras con valores similares a los del colágeno fresco (Van Klinken, 1999, Bocherens et al., 2005a), como es el caso de las dos muestras analizadas.

c) La relación atómica $\mathrm{C} / \mathrm{N}$ en el colágeno. En colágeno fresco esta relación fluctúa entre 2,9 y 3,6 y esta es la proporción que debe presentar el colágeno fósil para que se pueda considerar bien preservado (De Niro, 1985). Nuevamente, como se observa en la Tabla 1, el criterio de calidad se cumple en las dos muestras estudiadas.

\subsubsection{Consideraciones sobre las fuentes de variación de los valores isotópicos}

Para la interpretación de los valores obtenidos en el estudio isotópico del colágeno de ambos individuos es necesario tener en cuenta cuáles son los rangos de valores de las fuentes alimentarias y cómo éstos varían según distintos parámetros ambientales.

La composición isotópica relativa del carbono $\left(\delta^{13} \mathrm{C}\right)$ en los vegetales, como base de la cadena trófica, depende directamente del tipo de plantas. En el medio terrestre hay dos grupos principales (plantas C3 y plantas C4) que se diferencian en sus rutas fotosintéticas y por lo tanto en la manera de incorporar el carbono a sus tejidos. Las plantas toman el carbono del aire en forma de $\mathrm{CO}_{2}$ durante la fotosíntesis. $\mathrm{El} \mathrm{CO}_{2}$ del aire presenta valores isotópicos $\delta^{13} \mathrm{C}$ en torno al $-8 \%$. Las plantas, al tomar ese $\mathrm{CO}_{2}$, discriminan en contra del isótopo pesado, por lo que sus valores isotópicos estarán en general empobrecidos con respecto al $\mathrm{CO}_{2}$ del aire. Sin embargo, en la incorporación de ese carbono existe una diferencia importante entre las plantas C3 (especies arbóreas y arbustivas de cualquier zona, y herbáceas de climas templados) y las C4 (herbáceas tropicales, entre ellas el maíz, la caña de azúcar o el mijo), de manera que las primeras presentan valores de $\delta^{13} \mathrm{C}$ entre $-25 \%$ y $-28 \%$, mientras que los valores en las segundas fluctúan entre $-14 \%$ y $-12 \%$, aproximadamente (O'Leary, 1981; Van der Merwe, 1982).

Además, ciertos parámetros ambientales como la densidad de la cubierta arbórea, la temperatura y la humedad del medio también influyen en los valores isotópicos del carbono en los vegetales (Bocherens y Drucker, 2003a; Drucker et al., 2008). Los valores isotópicos de los vegetales se reflejan a su vez en los tejidos de los herbívoros, en los que el fraccionamiento isotópico produce un enriquecimiento en el isótopo pesado con respecto a la dieta, aunque las diferentes rutas de síntesis de esos tejidos producen valores diferentes según el tejido analizado. El colágeno óseo responde a las variaciones observadas en proteínas y su enriquecimiento final con respecto a los vegetales de la dieta es de aproximadamente el 5\% (Ambrose y Norr, 1993). Posteriormente en cada paso de la cadena trófica la variación es de aproximadamente $+1 \%$.

La composición relativa de los isótopos del nitrógeno $\left(\delta^{15} \mathrm{~N}\right)$ en el colágeno de los mamíferos se relaciona directamente con su posición en el seno de la cadena alimentaria, pues cada nivel trófico sobre el de herbívoro 
se caracteriza por un incremento en promedio para el valor de $\delta^{15} \mathrm{~N}$ de aproximadamente un 3-4\%o (Bocherens y Drucker, 2003a).

Los principales factores que afectan a la composición isotópica del nitrógeno en el colágeno de los mamíferos herbívoros son tres: la síntesis de nitrógeno en el suelo, la dieta del animal y el metabolismo del nitrógeno en el animal (Koch, 1998). La síntesis del nitrógeno en el suelo depende fundamentalmente de factores climáticos, y por lo tanto puede variar cronológica y geográficamente.

Las plantas que fijan el nitrógeno (fundamentalmente las leguminosas, familia Fabaceae) tienen valores de $\delta^{15} \mathrm{~N}$ similares a la composición del $\mathrm{N}_{2}$ atmosférico $(\sim 0 \%)$, mientras que aquellas que no asimilan este elemento por sí mismas y usan otras fuentes de nitrógeno (iones $\mathrm{NH}_{4}+$ y $\mathrm{NO}_{3}$ del suelo) suelen mostrar un rango más amplio de valores de $\delta^{15} \mathrm{~N}$, en general más positivos. En consecuencia, los animales que consumen plantas fijadoras de $\mathrm{N}_{2}$ mostrarán normalmente valores de $\delta^{15} \mathrm{~N}$ comprendidos entre $0 \%$ y $+4 \%$, mientras que en los herbívoros que se alimentan de plantas que no fijan el $\mathrm{N}_{2}$ este rango oscila entre el $+2 \%$ o y el $+8 \%$.

El medio marino representa un ecosistema diferente al terrestre, y las diferencias se reflejan también en los valores isotópicos. Los isótopos del carbono varían entre el medio continental (incluyendo el terrestre y aguas dulces) y el marino, en el que los valores de $\delta^{13} \mathrm{C}$ son más positivos, ya que la fuente de carbono para los productores primarios marinos no es el $\mathrm{CO}_{2}$ del aire sino principalmente carbono inorgánico disuelto, con señales isotópicas cercanas a 0\%o (Peterson \& Fry, 1987). Por ello los valores isotópicos de las faunas de medios oceánicos presentan valores de $\delta^{13} \mathrm{C}$ marcadamente menos negativos que los de medios continentales ya sean terrestres o de aguas dulces (Chisholm et al., 2006). Además, en el medio acuático (tanto marino como aguas continentales) las redes tróficas son mucho más complejas que en los ecosistemas terrestres, por lo que tanto los productores primarios como los sucesivos consumidores presentan valores de $\delta^{15} \mathrm{~N}$ mucho más elevados (Schoeninger et al., 1983; Schoeninger \& DeNiro, 1984) Así, la ingesta de alimentos de origen marino, de aguas continentales o puramente terrestre se puede reconocer con facilidad en los valores de ambos isótopos.

\subsubsection{Interpretación de los resultados isotópicos}

Los humanos, fósiles o actuales, suelen presentar señales isotópicas elevadas, fruto de su alimentación mixta. Sin embargo a lo largo de la historia de la humanidad se observan unas variaciones características en las señales isotópicas que reflejan la tendencia alimentaria en cada momento. Durante el Paleolítico la dieta va evolucionando de predominantemente carnívora en Neandertales (Bocherens \& Drucker 2003b; Bocherens et al., 2005b; Richards \& Trinkaus, 2009) a incluir alimentos de origen marino, al menos en las zonas costeras, desde finales del paleolítico superior (Richards et al., 2001, 2005; Drucker \& Bocherens 2004). La tendencia se invierte en el Neolítico con la expansión de la agricultura, cuando la alimentación a base de cereales y legumbres hace disminuir principalmente los valores isotópicos del nitrógeno (Tauber, 1981; Richards et al., 2003; Jay, 2008). Durante la romanización 
se observa en muchos yacimientos una tendencia, a veces muy marcada, al consumo de alimentos marinos en zonas incluso alejadas de los ambientes costeros (Richards et al., 1998; Prowse et al., 2004; Suárez Piñeiro, 2004; Serrulla et al., 2011).

A partir de unas señales isotópicas dadas es difícil reconstruir la dieta de un ser humano, debido a la enorme variedad de alimentos que puede llegar a consumir. En la Figura 2 se representan las señales isotópicas que registraría el colágeno humano a partir de diversos tipos de alimentos, si se siguieran dietas puras. Se han representado también los valores correspondientes a los individuos estudiados (MAC-110 y MAC-4414).

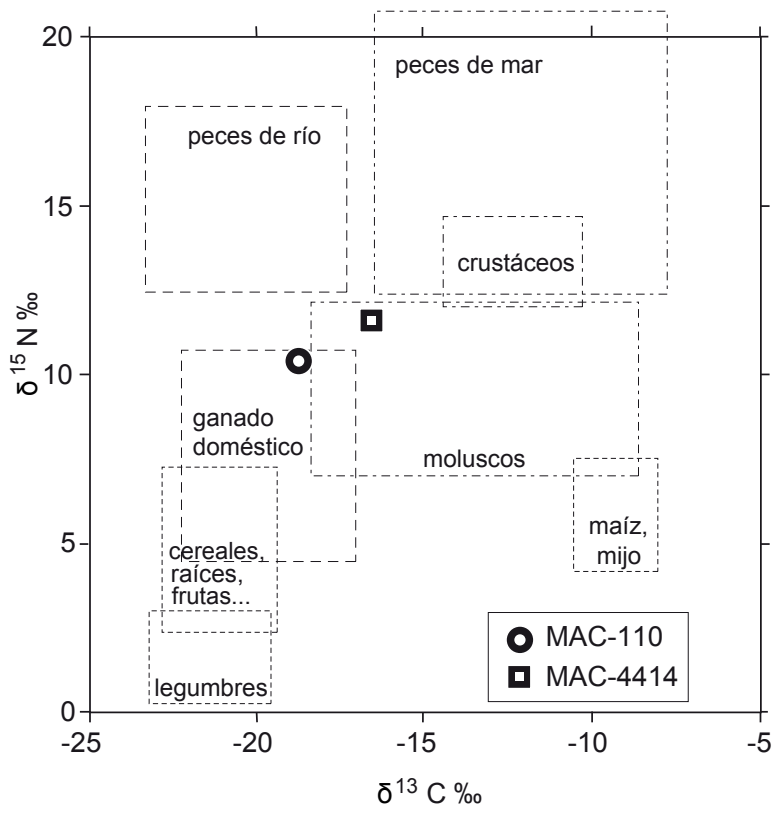

Fig. 2. Señales isotópicas que se producirían en el colágeno humano a partir de diversos tipos de alimentos, si se siguieran dietas puras (los valores ya están corregidos para el fraccionamiento isotópico correspondiente al colágeno óseo y a variaciones temporales en el carbono atmosférico). Se han representado también los valores correspondientes a los individuos estudiados (MAC110 y MAC-4414). Los datos isotópicos de diversos alimentos de origen terrestre y marino proceden de la literatura (Richards \& Hedges, 1999; Tykot 2003, Grall et al. 2006; Le Loc'h et al. 2008).
Los valores isotópicos obtenidos son totalmente congruentes con la cronología de los restos y su localización geográfica. Ambos individuos presentan valores que podrían considerarse intermedios, con una proporción apreciable de alimentos de origen marino en su dieta. Los valores de $\delta^{13} \mathrm{C}$ de MAC-4414 están más desplazados hacia las fuentes de origen marino, aunque sus valores de $\delta^{15} \mathrm{~N}$ son bajos para poder inferir una alimentación rica en pescado.

Los alimentos de origen vegetal tienen un menor aporte proteico que los de origen animal, por lo que su contribución a la dieta mediante el estudio del colágeno puede quedar enmascarada (Schoeller, 1999). Sin embargo, las legumbres sí tienen una proporción elevada de proteínas, lo que unido a sus característicos valores de $\delta^{15} \mathrm{~N}$ cercanos a cero permitirían reconocer claramente su consumo en proporciones altas. En la dieta de los dos individuos estudiados no parece que las legumbres hayan tenido un papel sustancial.

Sin embargo, el rango de valores posible de los diferentes grupos de alimentos puede ser 
excesivamente amplio para dar una idea más concreta del tipo de alimentos consumidos por los individuos estudiados. Con el fin de aproximarnos más a una caracterización de los alimentos habituales, hemos realizado un estudio comparativo con otras grupos humanos aproximadamente contemporáneos en localizaciones geográficas equivalentes (zonas costeras). Para este estudio comparativo incluimos valores isotópicos tomados de la bibliografía, procedentes de diversos yacimientos cuyos datos se ofrecen en la tabla 2. El resultado de esta comparación se puede observar en la Figura 3.
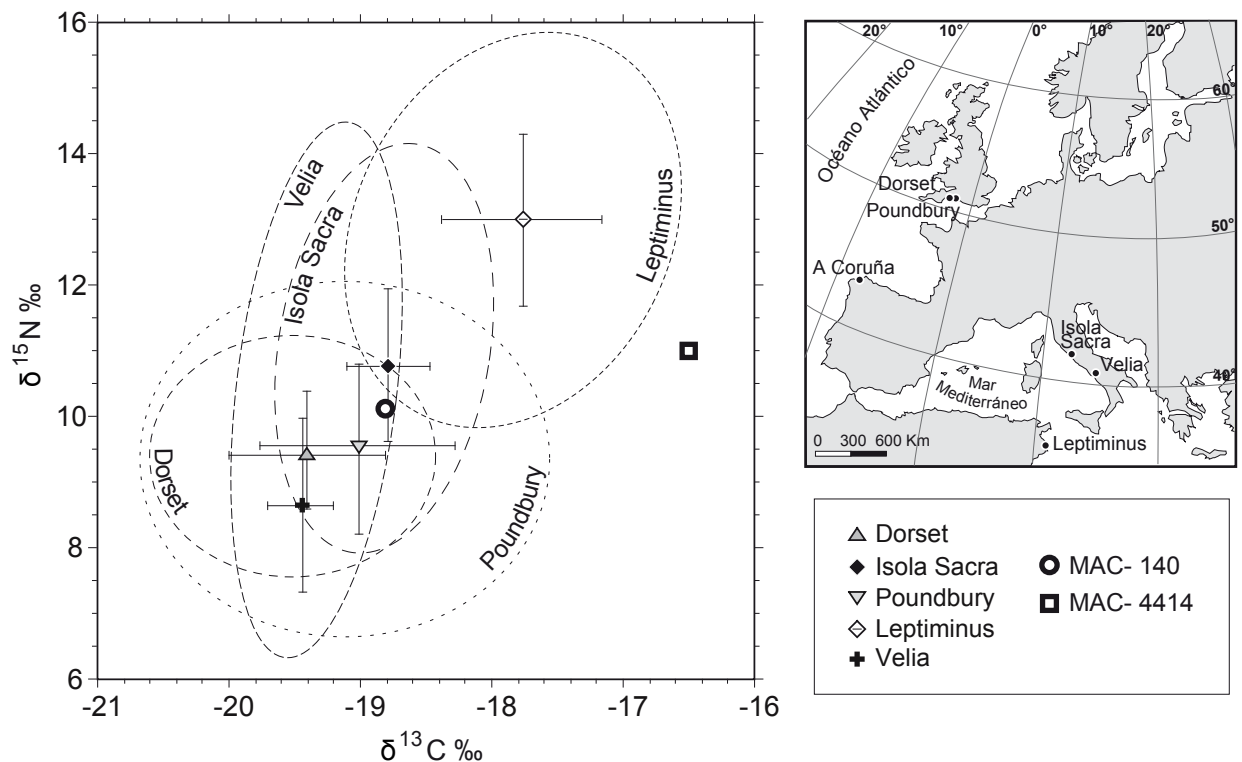

$\triangle$ Dorset

- Isola Sacra

$\nabla$ Poundbury

$\diamond$ Leptiminus

+ Velia

Fig. 3. Valores isotópicos de los individuos MAC-110 y MAC-4414 y otras poblaciones de edad romana tomadas de la literatura (Ver Tabla 2).

\begin{tabular}{|c|c|c|c|c|}
\hline Necrópolis & Localización & Edad & $\begin{array}{c}\text { Tipo de alimentación } \\
\text { inferida }\end{array}$ & Bibliografía \\
\hline Isola Sacra & Ostia, Italia & siglo II AD & $\begin{array}{c}\text { mixta, con abundan- } \\
\text { cia de pescado }\end{array}$ & Prowse et al., 2004 \\
\hline Velia & Sur de Italia & siglos I a II AD & $\begin{array}{c}\text { mixta, con menor } \\
\text { consumo de pescado }\end{array}$ & Craig et al., 2009 \\
\hline Leptiminus & Túnez & Siglos II a V AD & $\begin{array}{c}\text { muy rica en pescado, } \\
\text { en proporción cre- } \\
\text { ciente }\end{array}$ & $\begin{array}{c}\text { Keenleyside et al., } \\
2009\end{array}$ \\
\hline $\begin{array}{c}\text { Dorset (diversos } \\
\text { yacimientos) }\end{array}$ & $\begin{array}{c}\text { Costa sur de Gran } \\
\text { Bretaña }\end{array}$ & Siglo II a V AD & $\begin{array}{c}\text { Aporte de pescado } \\
\text { escaso }\end{array}$ & $\begin{array}{c}\text { Redfern et al., } \\
2010\end{array}$ \\
\hline Poundbury Camp & $\begin{array}{c}\text { Costa sur de Gran } \\
\text { Bretaña }\end{array}$ & Siglo IV AD & $\begin{array}{c}\text { Aporte de pescado } \\
\text { importante }\end{array}$ & $\begin{array}{c}\text { Richards et al., } \\
1998\end{array}$ \\
\hline
\end{tabular}

Tabla 2. Datos de los yacimientos de cronología similar con estudios de paleodieta mediante isótopos estables utilizados como referencia en este estudio. 
La muestra de Isola Sacra procede de una necrópolis romana del siglo II situado en la costa mediterránea. Estos romanos costeros muestran una alimentación mixta con abundancia de productos de origen marino (Prowse et al., 2004). En Velia, localidad situada unos $400 \mathrm{~km}$ al sur de Isola Sacra, pero también en la costa, se ha observado un rango mayor de tipos de alimentos, pero en general un menor consumo de pescado, como indican sus bajos valores medios de $\delta^{15} \mathrm{~N}$ (Craig et al., 2009). Los datos de Leptiminus (Túnez), de cuatro necrópolis de edades comprendidas entre los siglos II y V indican un consumo de pescado elevado y creciente en el tiempo (Keenleyside et al., 2009).

En la zona atlántica se han realizado estudios en algunos poblados romanos de Gran Bretaña, entre los que incluimos los de Dorset (Redfer et al., 2010) y Poundbury (Richards et al., 1998). La principal diferencia observada entre estos conjuntos y los mediterráneos radica en una marcada diferencia en los valores isotópicos del carbono, más negativos en los yacimientos británicos, que se ha achacado a una diferencia de entre 1 y 2 $\%$ ya en la base de la cadena trófica entre las zonas situadas más al sur de Europa y las más norteñas (Richards \& Van Klinken, 1997; Heaton, 1999). Además, los valores de $\delta^{15} \mathrm{~N}$ de los yacimientos ingleses no alcanzan valores tan elevados como los más altos de Isola Sacra o los del siglo V de Leptiminus. Sin embargo los valores de Poundbury son interpretados como reflejo de una alimentación con cierta proporción de alimentos de origen marino, sobre todo cuando se comparan con los obtenidos a partir de individuos del mismo yacimiento $\mathrm{u}$ otros cercanos, pero de la Edad del
Hierro, cuyos valores isotópicos muestran una elevada proporción de alimentos vegetales y ganadería, sin apenas indicios de consumo de pescado de mar (Richards et al., 1998; Jay, 2008). En los yacimientos de Dorset la diferencia en aporte de pescado entre los pobladores de la edad del Hierro y los romanizados apenas es notoria (Redfer et al., 2010).

El individuo MAC-110 presenta unos valores isotópicos intermedios entre los valores medios de Isola Sacra y Poundbury. Podríamos suponer una alimentación con poco aporte vegetal, mientras que la proteína animal provendría de la carne y alimentos marinos en un nivel bajo de la escala trófica, como sería el caso de los moluscos.

MAC-4414 presenta un valor de $\delta^{13} \mathrm{C}$ desplazado hacia valores menos negativos, y queda fuera de las categorías dietarias observadas en los yacimientos de edad romana. Podría suponerse que el desplazamiento en los valores de $\delta^{13} \mathrm{C}$ estaría relacionado con el consumo de plantas $\mathrm{C} 4$, como el mijo, pero sus valores de $\delta^{15} \mathrm{~N}$ son demasiado elevados, además de que el mijo no es un cultivo corriente en la Galicia romana (Suárez Piñeiro, 2004). De la combinación de valores isotópicos podemos deducir más bien una abundancia de moluscos en la dieta, como fue observada en algunas poblaciones históricas costeras (Tauber, 1986; Kosiba et al., 2007).

El consumo de moluscos como complemento alimentario está bien documentado en los yacimientos prehistóricos, sobre todo desde el Mesolítico. La presencia de numerosos concheros en localidades costeras es un ejemplo de este consumo (Bailey, 1978; Richards \& Hedges, 1999). Los moluscos suponen una fuente de proteína de digestibilidad 
similar a la carne, son ricos en aminoácidos esenciales, en ácidos grasos insaturados (Omega 3 y 6), en vitaminas B6 y B12 y en diversos oligoelementos (Belitz et al., 2009). Sin embargo, su escasísima biomasa y su pequeño aporte calórico (según Bailey, 1978, para conseguir el mismo aporte que un herbívoro de talla media se necesitan varias decenas de miles de moluscos) los hacen inviables como fuente principal de proteínas, además del elevado coste energético que supone su recolección.

\subsection{Integración de resultados antropológicos y de paleodieta con los datos arqueológicos}

A partir de los resultados del estudio antropológico y del estudio de la paleodieta, es posible interpretar que los individuos estudiados obtenían buena parte de sus recursos alimentarios a partir de moluscos, sin que probablemente sus necesidades nutritivas quedasen cubiertas. Los moluscos podrían ser obtenidos por los propios individuos, ya que las zonas adecuadas para su recolección abundan en las inmediaciones de la ciudad. Las patologías observadas en los esqueletos (hiponutrición, esfuerzo físico, golpes) podrían apoyar esta hipótesis.

Desde esta perspectiva es interesante, aunque complejo, llegar a una aproximación a la clase social a la que pudieran pertenecer estos restos humanos. Para la Coruña romana alto imperial (ss. I - II d. C.) contamos con diversos elementos epigráficos (aras, pedestales de estatuas, placas funerarias) (Pereira Menaut, 1991) que nos hablan de una población muy romanizada, posiblemente con elementos foráneos muy latinizados -están documentados libertos imperiales (Rodríguez Colmenero, 2005)- en lo que su onomástica refleja, haciéndonos pensar en un enclave fundado "ex novo" (Pérez Losada, 2002) en relación con el puerto y el faro, sin sustrato indígena. Para la ciudad bajo imperial tenemos muchos menos datos; no contamos con epigrafía y la retracción física de la ciudad parece indicar que se readapta el asentamiento a consecuencia, también, de una progresiva disminución de la vida comercial en paralelo a la pérdida de influencia del imperio en un territorio tan alejado de la metrópoli.

El lugar de donde proceden los tres esqueletos (ver figura 1) está relacionado con la única necrópolis romana conocida de la ciudad, aunque se sospeche que hubiera podido existir otra, por lo menos, en la zona del Campo de la Estrada. Está situada, como era común en las ciudades romanas, en uno de sus caminos de acceso, a su entrada, lo que debió constituir la actual calle Real. Posiblemente, en un principio se limitó a esa zona exterior (aproximadamente a la mitad de la c/ Real), pero posteriormente, al recogerse la ciudad romana hacia la actual Ciudad Vieja, esta cementerio se extendió sobre zonas antes habitadas (tramo inicial de la calle Real, Riego de Agua, Franja...). Suponemos que aquí se enterraba toda la población, sin que podamos discernir diferencias sociales hasta el momento, aunque sí haya algún indicio para su primera fase de existencia de alguna construcción funeraria por la aparición de placas y lápidas, sin que sepamos ni siquiera de que tipo, construcciones que podrían indicar alguna preeminencia en este aspecto.

En el marco físico de la ciudad bajo imperial, se testimonia la existencia de "villas", lo que podría hacer pensar, además de la existencia de una élite "señorial", en un proceso de ruralización de la sociedad, con un mayor peso del entorno como base 
de aprovisionamiento con respecto a lo que pudiese ser obtenido por vías comerciales, aunque ejemplos del mantenimiento de este intercambio no falten (López Pérez, 2010b). Esto crearía una población ligada para su sustento a los recursos naturales que están a su alcance. Son frecuentes en la ciudad, incluida la calle Real, los hallazgos de concheros de este momento (Vázquez Gómez, 1996, Pérez Losada, 2002). Las especies registradas con más frecuencia son ostra (Ostrea sp.), berberecho (Cardium sp.) lapa (Patella sp.) y mejillón (Mytilus sp.), aunque no necesariamente por ese orden pues no se hizo estudio cuantitativo. Corresponden a ambientes intermareales tanto de playa como rocosos, ambos plenamente accesibles en el entorno inmediato de la ciudad.

\section{CONCLUSIONES}

El trabajo multidisciplinar es esencial para mejorar el conocimiento en muchas áreas de la ciencia y por supuesto también en la Historia. La aplicación de métodos forenses al campo de la Prehistoria o de épocas históricas con escasez de fuentes documentales escritas permite obtener un nuevo punto de vista que ayuda a profundizar en detalles hasta ahora no demasiado explorados.

Los resultados de los estudios de paleodieta practicados en este trabajo confirman los datos arqueológicos, que señalan la presencia de moluscos en las excavaciones arqueológicas donde se hallaron estos esqueletos. Las patologías observadas son compatibles con el elevado esfuerzo físico que deberían haber realizado los individuos en caso de haber tenido que recolectar moluscos y otros alimentos de origen marino para su consumo personal. Las fuentes arqueológicas e históricas también indican una disminución de la vida comercial y un aumento de la vinculación a los recursos del entorno en la época bajo imperial en que vivieron los individuos estudiados. Todos los datos coinciden con el empobrecimiento generalizado del entorno urbano y la calidad de vida en Brigantium desde el Siglo IV, en contraste con el auge de siglos anteriores.

\section{AGRADECIMIENTOS}

Este estudio fue financiado por el Excelentísimo Ayuntamiento de A Coruña para la exposición "Rostros Brigantium" (diciembre de 2008-Abril de 2009), con motivo del $8^{\circ}$ aniversario de la fundación de la ciudad. Se agradece especialmente al Director del Museo Arqueológico Municipal, D. José María Bello Diéguez, la cesión del material para su estudio y exposición. Así mismo, agradecemos la colaboración de Benito López de Abajo, José Alfeirán, José Manuel Coello, Cruz Galindo, Enrique Pérez, el personal del Servicio de Radiología del Hospital Fundación Pública Verin, Iago Martínez y Prudencio García. El estudio isotópico se integra en la red CONSILIENCIA (R2014/001) del Programa de Consolidación e Estructuración Redes de la Xunta de Galicia.

\section{BIBLIOGRAFÍA}

Ambrose, S.H. 1990. Preparation and characterization of bone and tooth collagen for stable carbon and nitrogen isotope análisis. Journal of Archaeological Science 17: 431-451. 
Ambrose, S.H. y Norr, L. 1993. Experimental evidence for the relationship of the carbon isotope ratios of whole diet and dietary protein to those of bone collagen and carbonate. In: J. Lambert y G. Grupe (eds.): Prehistoric Human Bone. Archaeology at the Molecular Level. Springer Verlag. Berlín: 1-37.

Bailey, G.N. 1978. Shell middens as indicators of post-glacial economics: a territorial perspective. In: P. Mellars (ed.): The Early Post-Glacial settlement of Northern Europe. Duckworth. London: 37-63.

Belitz, H.-D.; Grosch, W. y Schieberle, P. 2009. Food Chemistry. Springer. Berlín. $1070 \mathrm{pp}$.

Bello Diéguez, J. M. 1995. Historia de La Coruña. Editorial Vía Láctea. A Coruña. $456 \mathrm{pp}$.

Bello Diéguez, J.M. 2009. Brigantium y su faro. Contextos arqueológicos en la ciudad de A Coruña. In: Arias Vilas, F., Fernández Ochoa, C., Morillo, A., (eds.): Torre de Hércules: finis terrae lux. Simposio sobre os faros romanos e a navegación occidental na antiguidade. Brigantium, 20, 43-66, A Coruña.

Bocherens, H. y Drucker, D. 2003a. Trophic level isotopic enrichments for carbon and nitrogen in collagen: case studies from recent and ancient terrestrial ecosystems. International Journal of Osteoarchaeology 13: 46-53.

Bocherens, H. y Drucker, D. $2003 \mathrm{~b}$. Reconstructing Neandertal diet from 120,000 to $30,000 \mathrm{BP}$ using carbon and nitrogen isotopic abundances. In: M. Patou-Mathis y H. Bocherens (eds.): Le rôle de l'environnement dans les comportements des chasseurs-cueilleurs préhistoriques. British Archaeological
Reports, International Series 1105, Archaeopress. Oxford: 1-7.

Bocherens, H.; Billiou, D.; Patou-Mathis, M.; Bonjean, D.; Otte, M. y Mariotti, A. 1997. Palaeobiological implications of the isotopic signatures $\left({ }^{13} \mathrm{C},{ }^{15} \mathrm{~N}\right)$ of fossil mammal collagen in Scladina Cave (Sclayn, Belgium). Quaternary Research 48: 370-380.

Bocherens, H.; Drucker, D.; Billiou, D. y Moussa, I. 2005a. Une nouvelle approche pour évaluer l'état de conservation de l'os et du collagène pour les mesures isotopiques (datation au radiocarbone, isotopes stables du carbone et de l'azote). L'Anthropologie 109: 557-567.

Bocherens, H.; Drucker, D. G.; Billiou, D.; Patou-Mathis, M. y Vandermeersch, B. 2005b. Isotopic evidence for diet and subsistence pattern of the Saint-Césaire I Neanderthal: Review and use of a multi-source mixing model. Journal of Human Evolution 49: 71-87.

Campillo, D. 1983. La enfermedad en la prehistoria. Introducción a la paleopatología. Salvat. Barcelona.

Campillo, D. 1993. Paleopatología: los primeros vestigios de la enfermedad. Fundación Uriach 1838. Barcelona.

Chisholm, B.S.; Nelson, D.E. y Schwarcz, H.P. 2006. Stable carbon isotope ratios as a measure of marine versus terrestrial protein in ancient diets. Science 216: 1131-1132.

Craig, O.E.; Biazzo, M.; O'Connell, T.C.; Garnsey, P.; Martinez-Labarga, C.; Lelli, R.; Salvadei, L.; Tartaglia, G.; Nava, A.; Reno, L; Fiammenghi, A.; Rickards, O. y Bondioli, L. 2009. Stable Isotopic Evidence for Diet at the 
Imperial Roman Coastal Site of Velia (1st and 2nd Centuries AD) in Southern Italy. American Journal of Physical Anthropology 139: 572-583.

Dastugue, J. y Gervais, V. 1992. Paleopathologie du squelette humain. Ed. Boubée. París.

De Niro, M.J. 1985. Post-mortem preservation and alteration of in vivo bone collagen isotope ratios in relation to paleodietary reconstruction. Nature 317: 806-809.

Drucker, D.G. y Bocherens, H. 2004. Carbon and nitrogen stable isotopes as tracers of diet breadth evolution during Middle and Upper Palaeolithic in Europe. International Journal of Osteoarchaeology 14 (3-4): 162-177.

Drucker, D.; Bridault, A.; Hobson, K.; Szuma, E. y Bocherens, H. 2008. Can carbon-13 in large herbivores reflect the canopy effect in temperate and boreal ecosystems? Evidence from modern and ancient ungulates. Palaeogeography, Palaeoclimatology, Palaeoecology 195: 375-388.

Grall, J.; Le Loc'h, F.; Guyonnet, B. y Riera, P. 2006. Community structure and food web based on stable isotopes $\left(\delta^{15} \mathrm{~N}\right.$ and $\left.\delta^{13} \mathrm{C}\right)$ analysis of a North Eastern Atlantic maerl bed". Journal of Experimental Marine Biology and Ecology 338 (1): 1-15.

Heaton, T.H.E., 1999. Spatial, species and temporal variations in the ${ }^{13} \mathrm{C} /{ }^{12} \mathrm{C}$ ratios of C3 plants; implications for palaeodiet Studies. Journal of Archaeological Science 26: 637-644.

Hedges, R.; Stevens, R.E. y Richards, M.P. 2004. Bone as a stable isotope archive for local climatic information. Quaternary Science Review 23: 959-965.
Hedges, R.E.M. y Reynard, L.M. 2007. Nitrogen isotopes and the trophic level of humans in archaeology. Journal of Archaeological Science 34: 1240-1251.

Janz, R.L.; Ousley, S.D. y Moore-Jansen P.M. 1994. Data collection procedures for forensic skeletal material. Report of investigations $\mathrm{n}^{\circ} 48$. Departament of Anthropology. University of Tennessee. Knoxville.

Jay, M. 2008. Iron Age diet at Glastonbury Lake Village: the isotopic evidence for negligible aquatic resource consumption. Oxford Journal of Archaeology 27 (2): 201-216.

Keenleyside, A.; Schwarcz, H.; Stirling, L. y Ben Lazreg, N. 2009. Stable isotopic evidence for diet in a Roman and Late Roman population from Leptiminus, Tunisia. Journal of Archaeological Science 36: 51-63.

Koch, P.L. 1998. Isotopic reconstruction of past continental environments. Annual Review of Earth and Planetary Sciences 26: 573

Kosiba, S.B.; Tykot, R.H. y Carlsson, D. 2007. Stable isotopes as indicators of change in the food procurement and food preference of Viking Age and Early Christian populations on Gotland (Sweden). Journal of Anthropological Archaeology 26 (3): 394-411.

Le Loc'h, F.; Hily, C. y Grall, J. 2008. Benthic community and food web structure on the continental shelf of the Bay of Biscay (North Eastern Atlantic) revealed by stable isotopes analysis. Journal of Marine Systems 72: 17-34.

López Pérez, C. 2010a. Reflexiones sobre la época Flavia en Brigantium a partir de los datos proporcionados por la Terra Sigillata. CuPAUAM 36, 95-106. 
López Pérez, C. 2010b. La vajilla fina de mesa de época romana en los solares $n^{\circ}$ 10-12 de la calle Tabernas (A Coruña). Gallaecia 29: 191-202.

Lubell, D.; Jackes, M.; Schwarcz, H.; Knyf, M. y Meiklejohn, C. 1994. The Mesolithic-Neolithic transition in Portugal: isotopic and dental evidence of diet. Journal of Archaeological Science 21, 201-216.

Luengo, J.M. 1955. Excavaciones de la necrópolis romana de La Coruña. III Congreso Nacional de Arqueología (Santiago, 1953): 415-427. Zaragoza.

Mann, R.W.y Hunt, D.R. 2005. Photographic regional atlas of bone disease. A guide to pathologic and normal variation in the human skeleton. 2nd Edition. Charles C Thomas Publisher LTD Springfield. Illinois.

Naveiro López, J. L. 1994. El golfo Ártabro. Arqueología e historia del gran puerto de los Galaicos Lucenses. Asociación Amigos do Museo Arqueolóxico. Monografías urxentes, vol. 5. A Coruña, $96 \mathrm{pp}$.

Naveiro López, J.L. 1988. Arqueología urbana en La Coruña y definición del asentamiento romano. In: R. Villares Paz (coord.): La ciudad y el mundo urbano en la Historia de Galicia. Universidade de Santiago. Santiago de Compostela: 35-62.

Nossintchouck, R.M. 1991. Manuel d'odontologie médico-legale. Masson. París.

O’leary, M.H. 1981. Carbon isotope fractionation in plants. Phytochemistry 20 (4): 553-567.

Ortner, D.J. 2003: Identification of pathological conditions in human skeletal remains. 2nd Edition. Academic Press. San Diego.
Pereira Menaut, G. 1991. Corpus de inscripcións romanas de Galicia. Provincia de A Coruña. Consello da Cultura Galega. Santiago de Compostela.

Pérez Losada, F. 2002. Entre a cidade e a aldea. Estudio arqueohistórico dos "aglomerados secundarios" romanos en Galicia. Brigantium 13: 15-348.

Pérez, A. (ed.) 1995. Salud, enfermedad y muerte en el pasado. Consecuencias biológicas del estrés y la patología. Actas del III Congreso Nacional de Paleopatología (Barcelona, 1995). Barcelona.

Peterson, B.J. y Fry, B. 1987. Stable isotopes in ecosystem Studies. Annual Review of Ecology and Systematics 18: 293-320.

Prowse, T.; Schwarcz, H.P.; Saunders, S.; Macchiarelli, R. y Bondioli, L. 2004. Isotopic paleodiet studies of skeletons from the Imperial Roman-age cemetery of Isola Sacra, Rome, Italy. Journal of Archaeological Science 31: 259-272.

Quatrehomme, G., Balaguer, T.; Staccini, P. y Alunni-Perret, V. 2007. Assessment of the accuracy of three-dimensional manual craniofacial reconstruction: a series of 25 controlled cases. International Journal of Legal Medicine 121: 469-475.

Redfer, R.; Hamli, C. y Beavan Athfield, N. 2010. Temporal changes in diet: a stable isotope analysis of late Iron Age and Roman Dorset, Britain. Journal of Archaeological Science 37(6): 1149-1160.

Reichs KJ (ed.) 1998. Forensic Osteology. Advances in the identification of human remains. Second Edition. Charles C Thomas Publisher LTD Springfield. Illinois.

Reverte Coma, J.M. 1991. Antropología Forense. Ministerio de Justicia. Madrid. Richards, M.P. 2002. A brief review of the 
archaeological evidence for Palaeolithic and Neolithic subsistence. European Journal of Clinical Nutrition 56: 1270 1278.

Richards, M.P. y Hedges, R.E.M. 1999. Stable isotope evidence for similarities in the types of marine foods used by the late Mesolithic humans at sites along the Atlantic coast of Europe. Journal of Archaeological Science 26: 717-722.

Richards, M.P. y Trinkaus, E. 2009. Isotopic evidence for the diets of European Neanderthals and early modern humans. Procceedings of the National Academy of Sciences of the USA 106 (38): 1603416039.

Richards, M.P. y van Klinken, G.J. 1997. A survey of European human bone stable carbon and nitrogen isotope values. In: A.G.M. Sinclair, E.A. Slate y J.A.J. Gowlett (eds.): Archaeological Sciences, Oxbow Archaeological Monograph, Oxford: 363-368.

Richards, M.P.; Hedges, R.E.M.; Molleson, T.I. y Vogel, J. C. 1998. Stable isotope analysis reveals variations in human diet at the Poundbury Camp cemetery site. Journal of Archaeological Science 25: 1247-1252.

Richards, M.P.; Jacobi, R.; Cook, J.; Pettit, P.B. y Stringer, C. 2005. Isotope evidence for the intensive use of marine foods by Late Upper Palaeolithic humans. Journal of Human Evolution 49 (3): 390-394.

Richards, M.P.; Pettit, P.B.; Stiner, M.C. y Trinkaus, E. 2001. Stable isotope evidence for increasing dietary breadth in the European mid-Upper Paleolithic. Procceedings of the National Academy of Sciences of the USA 98 (11): 6528 -6532. Richards, M.P.; Price, T.D. y Koch, E. 2003. The mesolithic and neolithic subsistence in Denmark: new stable isotope data. Current anthropology 44 (2): 288-294.

Rodríguez Colmenero, A. 2005. Las nuevas stationes lucensis et brigantina en el finisterre ibérico del imperio romano. Palaeohispánica 5: 873-892.

Schoeller, D.A. 1999: Isotope fractionation: Why aren't what we eat?. Journal of Archaeological Science 26: 667-673.

Schoeninger, M. y DeNiro, M. 1984. Nitrogen and carbon isotopic composition of bone collagen from marine and terrestrial animals. Geochimica et Cosmochimica Acta 48: 625-639.

Schoeninger, M., DeNiro, M. y Tauber, H. 1983. Stable nitrogen isotope ratios of bone collagen reflect marine and terrestrial components of prehistoric human diet. Science 220: 1381-1383.

Serrulla, F. (coord.) 2001. Monografico Antropologia Forense. Boletin Galego de Medicina Legal e Forense $\mathrm{n}^{\circ} 10$. Asociación Galega de Médicos Forenses. Verín (Ourense).

Serrulla, F. y Gómez, M. 2008. Aplicaciones de la técnica de aproximación facial forense en la identificación humana individual. Cuadernos de Medicina Forense 53-54: 291-307.

Serrulla, F.; Grandal-d'Anglade, A.; Vilar Labarta, S. y Gómez, M. 2011. Aproximación facial y paleodieta en un esqueleto de la necrópolis de El Vergel (Ávila, España). Munibe (AntropologiaArkeologia) 62, 341-349.

Suárez Piñeiro, A.M. 2004. Sentados á mesa dun galaico-romano: dieta, alimentación e hábitos culinarios na Galicia romana. Gallaecia 23: 155-172.

Tauber, H. 1981. ${ }^{13} \mathrm{C}$ evidence for dietary habits of prehistoric man in Denmark. Nature 292: 332-333. 
Tauber, H. 1986. Analysis of stable isotopes in prehistoric populations. In: B. Hermann (ed.): Innovative Trends in Prehistoric Anthropology. Mitteilungen del Berliner Gesellshaft für Anthropologie, Ethnologie und Urgeschichte 7: 31-38.

Tieszen, L.L.; Boutton, T.W. 1989. Stable carbon isotopes in terrestrial ecosystem research. In: P.W. Rundel, J.R. Ehleringer y K.A. Nagy (eds.): Stable isotopes in ecological research. Springer Verlag, Berlín: 167-195.

Tomás Botella, V. 2004. Control arqueolóxico de obra en r/Real 34 (A Coruña). Informe valorativo (informe inédito).
Tykot, R.H. 2003. Stable isotopes and diet: You are what you eat. In: M. Martini, M. Milazzo y M. Piacentini (eds.): Proceedings of the International School of Physics "Enrico Fermi". IOS Press, Amsterdam: 433-444.

Van Der Merwe, N.J. 1982. Carbon isotopes, photosynthesis, and archaeology. American Scientist 70: 596-606.

Van Klinken, G.J. 1999. Bone collagen quality indicators for palaeodietary and radiocarbon measurements. Journal of Archaeological Science 26: 687-695.

Vázquez Gómez, X.L. 1996. Excavación en la calle de la Franja 9-11. A Coruña. Gallaecia 14-15: 411-461. 